\title{
Renal cancer seeding metastases following retroperitoneoscopic- assisted cryoablation: A case report
}

\author{
Maaike W. van de Kamp, MD,* Bettina Kortekaas, MD,; Brunolf W. Lagerveld, MD* \\ *Department of Urology, St. Lucas Andreas Hospital, Amsterdam, The Netherlands; "Department of Urology, Free University of Amsterdam, Amsterdam, The Netherlands
}

Cite as: Can Urol Assoc J 2015;(9-10):E641-3. http://dx.doi.org/10.5489/cuaj.2850

Published online September 9, 2015.

\section{Abstract}

Nephron-sparing laparoscopy is the standard surgical treatment for clinical T1a renal tumours. However, the laparoscopic technique brings in its specific oncological safety concerns. Seeding metastases are reported: peritoneal metastases, port-tract metastases, and (sub-) cutaneous metastases. The method of laparoscopic assisted renal mass cryoablation is marked by the fact that traumatic tumour tissue handling is unavoidable. This case report reviews the rare occasion of seeding metastases in the retroperitoneal space following laparoscopic cryoablation of a small renal mass. The primary tumour showed no focal recurrence as reported by histological examination. The combination of two events as harming the integrity of cancer tissue and gas-circulation leading to the development of metastases in the retroperitoneal cavity is discussed. The combination of iatrogenic harming cancer tissue integrity and $\mathrm{CO}_{2}$-circulation leads to metastases in the retroperitoneal cavity. Therefore, we recommend performing image-guided renal mass biopsies before considering cryoablative surgery.

\section{Introduction}

Laparoscopic cryoablation (LCA) is considered a valid alternative treatment for T1 a renal tumours. ${ }^{1}$ Comparison of laparoscopic partial nephrectomy versus laparoscopic cryoablation showed that cryoablation was associated with less blood loss and delayed complications. ${ }^{2}$ Laparoscopic cryoablation is recognized as a less technically advanced procedure with good results compared to partial nephrectomy. ${ }^{3}$ However, long-term data after laparoscopic cryoablation, including the development of metastases, are scarce.

Laparoscopic surgery is associated with better preservation of systemic immune responses compared to open surgery. However, there is evidence that the local immune response of the peritoneum is depressed following laparoscopy. ${ }^{4}$ Other factors, such as $\mathrm{CO}_{2}$-circulation, traumatic tumour tissue manipulation, and specimen extraction, might be related to the occurrence of seeding metastases.

\section{Case presentation}

In 2007, a 65-year-old woman was referred for laparoscopic cryoablation of a renal tumour measuring $21 \mathrm{~mm}$ (Fig. 1). Her medical history revealed a multinodular goiter, stage 1 melanoma cancer, and Caesarean section. The Charlson comorbidity score and performance score were both 0 . The anatomical complexity of the renal mass was defined by the RENAL nephrometry index scored: $1+2+2+2=7$, suffix ' $x .^{\prime 5}$ Chest X-ray showed no metastases.

The laparoscopic cryoablation was video recorded and reviewed. Via a small incision, we entered the retroperitoneal space and under continuous $\mathrm{CO}_{2}$-insufflation $(15 \mathrm{mmHg})$, retroperitoneoscopic surgery was assessed. Gerota's fascia was opened and the renal mass approached. We identified the tumour with endoscopic ultrasonography (Hitachi Medical Systems). Percutaneously, using a spring-loaded device (Magnum, Bard, Covington), we performed three 16-gauge core needle biopsies of the tumour, which resulted in minimal bleeding of the biopsied mass. Consecutively, 4 Iceseed cryoprobes (Galil Medical) were percutaneously introduced in the tumour. One temperature-probe was placed in the normal renal parenchyma just below the caudal rim of the tumour. Two cycles of 10-minute freezing were performed. After thawing, all probes were removed. No intra-operative complications, in particular no fracturing of the tumour, were recorded. Primary histology revealed a clear cell renal cell carcinoma, Fuhrman grade 1. The specimen was reviewed confirming the initial diagnosis and grading.

At follow-up, contrast-computed tomography (CT) showed no enhancement of the ablated lesion, a diminishing volume of the ablation area, and no renal mass de novo. However, at the 5-year follow-up, the contrast-CT showed several enhancing nodules $(3-7 \mathrm{~mm})$ in the peri-renal fat of the right kidney (Fig. 2). 


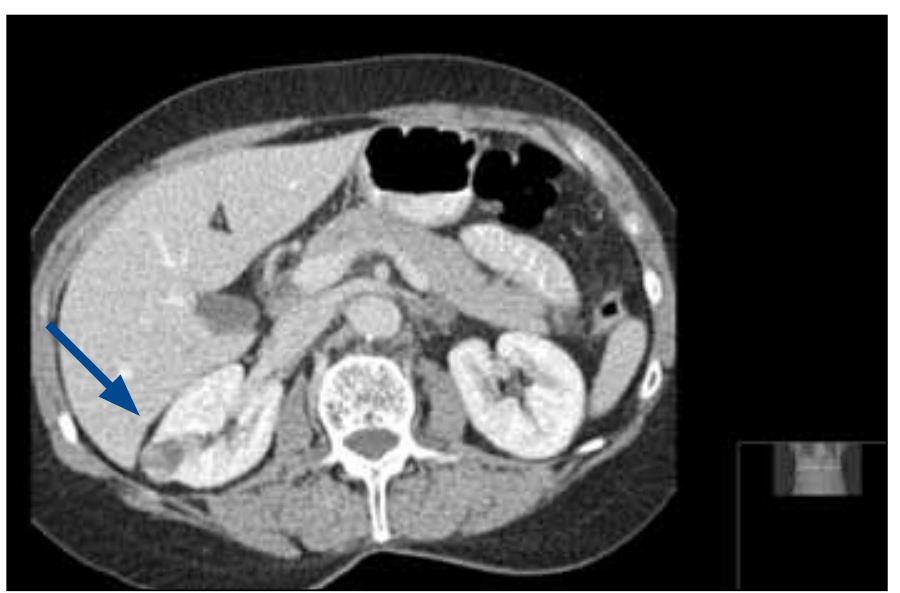

Fig. 1. Intravenous contrast abdominal computed tomography showing a contrast-enhancing solid lesion of $21 \mathrm{~mm}$ diameter (blue arrow) in the right kidney.

A chest-CT showed no metastases. A 18-Fluorodeoxyglucose positron emission tomography (FDGPET) revealed no pathologic metabolic activity (Fig. 3). However, at the repeated contrast-CT 6 months later, the lesions in the fat showed growth.

A radical laparoscopic nephrectomy was performed. The specimen contained tissue of the entire retroperitoneal space between the right iliac vessels, liver, inferior vena cava, and lateral abdominal wall. A single nodular lesion was dissected from the muscular psoas fascia (Fig. 4).

Histological examination demonstrated 8 metastases located in the peri-renal fat with a maximum size of $7 \mathrm{~mm}$ in diameter. Immunohistochemistry confirmed the diagnosis of clear cell carcinoma, without positive margins. The cryoablation zone revealed only fibrotic tissue. At the final imaging in July 2014, scans revealed no signs of recurrence.

\section{Discussion}

This case was reviewed to discriminate the event that could have contributed to the occurrence of seeding metastases. Therefore, viable tumour cells must be liberated from the primary tumour, be transported, and need a favorable environment for growth.

Performing a laparoscopic cryoablation, three possible events of traumatic tumour tissue manipulation are seen: (1) tumour from peri-renal fat; (2) tumour biopsy; and (3) cryoprobe placement and removal. All tumour manipulation is under continuous $\mathrm{CO}_{2}$ flow, working as a vehicle for viable cellular spread in the working cavity. The dissection of the plane is performed with a minimal risk for tumour tissue injury. There instead, intra-operative biopsies are harming the tumour integrity. Viable cancerous cells can be dislocated and spread in the $\mathrm{CO}_{2}$ circulated area. Also cryoprobe placement disrupts tumour integrity. However,

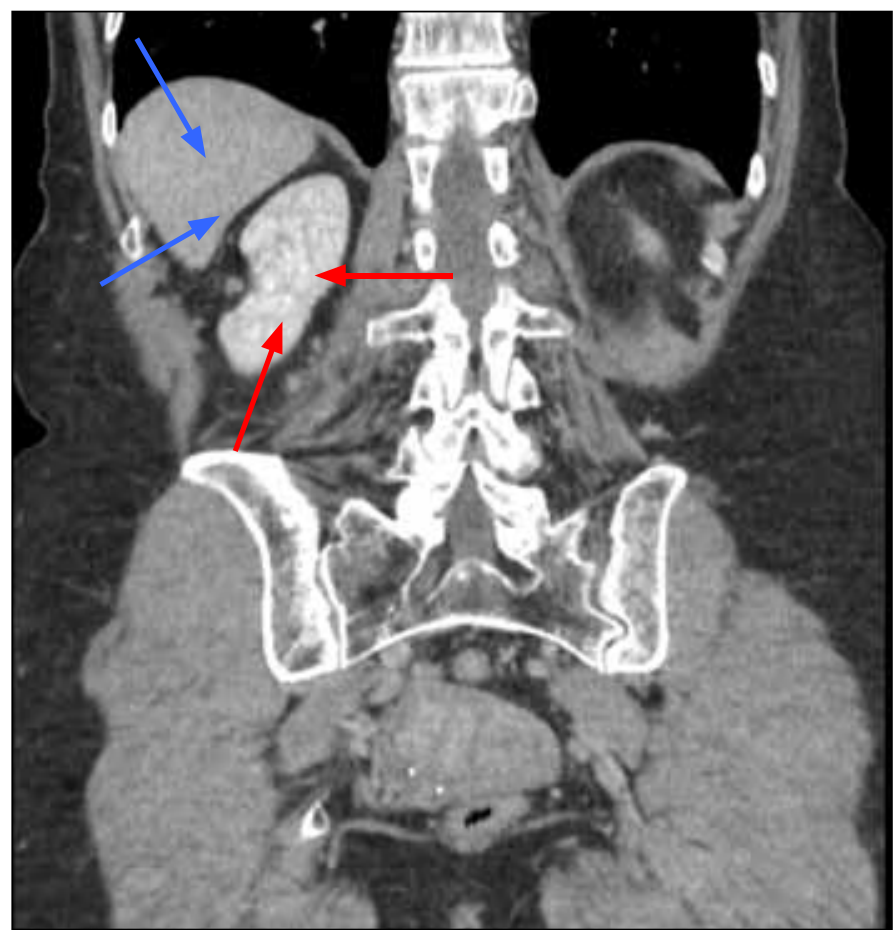

Fig. 2. Abdominal intravenous contrast computed tomography, at 5-year follow up after retroperitoneoscopic renal mass cryoablation. A non-contrast enhancing area, indicating the ablation zone, is shown in the lateral border of the renal capsule (blue arrows). Multiple solid nodular lesions are located in the perirenal fat (red arrows). Note that the ablation zone has created a dimple defect in the lateral contour of the kidney filled with non contrast-enhancing tissue. Within this tissue there is a contrast-enhancing nodule that is not attached to the renal capsule.

the introduced probes remain in situ for freezing purposes. The cryogenic physical conditions in the tissue nearest to the probes are such that no cells in that area are expected to survive a normal cryoablation procedure. Therefore, we postulate that, after removal of the cryoprobes, the leakage from probe insertion places consisting of melting ice crystals, non-vital cells and cellular debris will not result in seeding. Clearly, replacing cryoprobes before cryoablation is an equal risk factor in the spread of vital cells as the biopsies are performed.

Four cryobiological response mechanisms to clinical cryogenic ablation of tissues are recognized. ${ }^{6}$ The primary injury mechanisms are immediate and vascular cell injury, followed by apoptosis and immunological processes. These mechanisms of secondary response to cryogenic conditions are mostly at the outer rim of the ice-ball. Therefore, only cells that survived the direct injury conditions and are dislocated from this zone can inoculate. Theoretically, these cells can only dislocate after a tumour fractures.

Earlier data of clinical practice report the regression of metastatic lesions after cryoablation of the primary tumour that suggests a potential systemic response to the local cryoinjury. ${ }^{7}$ Sabel $^{8}$ describes the existing evidence of both stimu- 


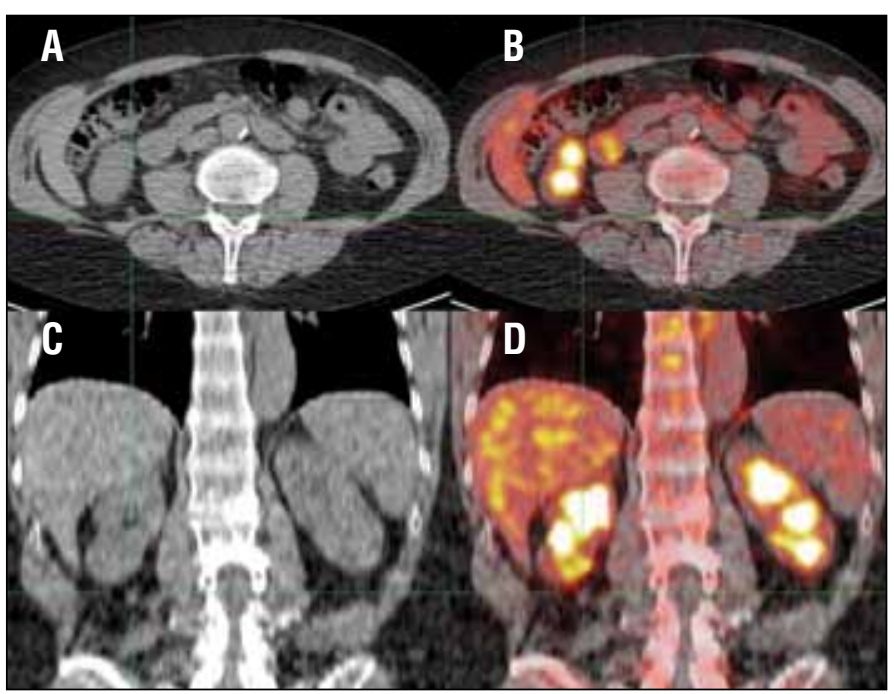

Fig. 3. An abdominal ${ }^{18} \mathrm{~F}$-fluordeoxyglucose positron emission tomography combined with low-dose CT. Panel A (axial) and B (coronal) show at the intersection of the green lines a nodule in the perirenal fat of the right kidney. Panel C (axial) and D (coronal) show the fusion of ${ }^{18} \mathrm{~F}-\mathrm{FDG}$ PET with lowdose CT. The nodular lesion (at the intersection of the green lines) shows no attenuation.

latory and suppressive immune responses after cryoablation. In addition to the cryo-induced immunological response, there is evidence that an immune response is associated to the laparoscopy itself that may trigger inoculation of cancer cells. ${ }^{4}$

The performance of intra-operative biopsies is common to the laparoscopic cryosurgery procedure. An earlier study showed that intra-operative biopsies avoid non-diagnostic results. ${ }^{9}$ However, the guidelines committee at the American Urological Association states that renal mass biopsy is mandatory before ablation. ${ }^{1}$ Furthermore, Volpe and colleagues reported that the complication-risk of image guided renal mass biopsies using coaxial techniques is low. ${ }^{10}$ The probability for seeding metastases related to traumatic tumour tissue manipulation, as with intra-operative biopsies, is an additional rationale in recommending biopsies before ablative surgery.

\section{Conclusion}

In this case of laparoscopic cryoablation, the combination of iatrogenic harming cancer tissue integrity and $\mathrm{CO}_{2}-$ circulation led to the development of metastases in the retroperitoneal cavity. Therefore, we recommend performing image-guided renal mass biopsies before considering cryoablative surgery.

Competing interests: The authors have no direct or indirect commercial incentive associated with publishing the manuscript. Brunolf W. Lagerveld proctors laparoscopic and image guided cryoablation of renal tumors on behalf of Galil Medical.

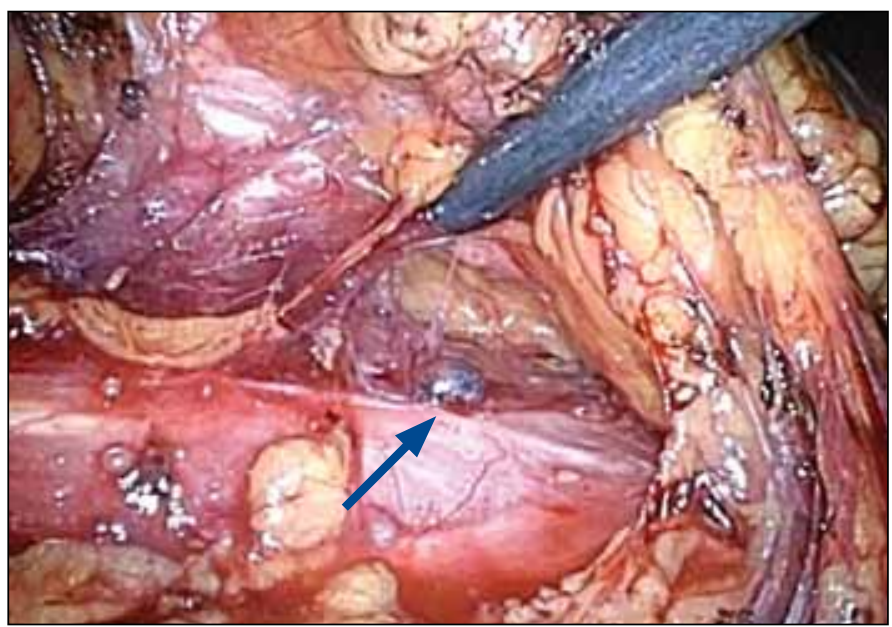

Fig. 4. Video-recorded image of the transperitoneal radical nephrectomy at the right side. Note one of the metastases at the muscular psoas (blue arrow).

Acknowledgements: We want to thank J.A. van der Zee, urologist at the St. Lucas Andreas Hospital (Amsterdam, the Netherlands) for his revisions to this manuscript.

This paper has been peer-reviewed.

\section{References}

1. Campbell SC, Novick AC, Belldegrun A, et al. Practice Guidelines Committee of the American Urological Association: Guideline for management of the clinical T1 renal mass. J Urol 2009;182:1271-79. http:// dx.doi.org/10.1016/i.juro.2009.07.004

2. Desai MM, Aron M, Gill IS. Laparoscopic partial nephrectomy versus laparoscopic cryoablation for the small renal tumor. Urology 2005;66:23-8. http://dx.doi.org/10.1016/j.urology.2005.06.114

3. Klatte $T$, Grubmüller $B$, Waldert $M$, et al. Laparoscopic cryoablation versus partial nephrectomy for the treatment of small renal masses: Systematic review and cumulative analysis of observational studies. Eur Urol 2011:60:435-43. http://dx.doi.org/10.1016/i.eururo.2011.05.002

4. Hegarty N, Dasgupta P. Immunological aspects of minimally invasive oncologic surgery. Curr Opin Urol 2008;18:129-33. http://dx.doi.org/10.1097/MOU.0b013e3282f517fc

5. Kutikov A, Uzzo RG. The R.E.N.A.L. nephrometry score: A comprehensive standardized system for quantitating renal tumor size, location and depth. J Urol 2009;182:844-53. http://dx.doi.org/10.1016/i. juro.2009.05.035

6. Gage A, Baust J. Mechanisms of tissue injury in cryosurgery. Cryobiology 1998;37:171-86. http:// dx.doi.org/10.1006/cryo.1998.2115

7. Soanes WA, Ablin RJ, Gonder MJ. Remission of metastatic lesions following cryosurgery in prostatic cancer: Immunological considerations. J Urol 1970;104:154-59.

8. Sabel MS. Cryo-immunology: A review of the literature and proposed mechanisms for stimulatory versus suppressive immune responses. Cryobiology 2009;58:1-11. http://dx.doi.org/10.1016/i. cryobiol.2008.10.126

9. Lagerveld BW, van Dekken H, van Leenders GJLH, et al. The role of pathology in small renal mass Iaparoscopic cryoablation. Adv Urol 2012;539648. hitrp://dx.doi.org/10.1155/2012/539648.

10. Volpe A, Finelli A, Gill IS, et al. Rationale for percutaneous biopsy and histologic characterization of renal tumors. Eur Urol 2012;62:491-504. http://dx.doi.org/10.1016/i.eururo.2012.05.009

Correspondence: Dr. B.W. Lagerveld, St. Lucas Andreas Hospital, Department of Urology, Jan Tooropstraat 164, 1006 AE Amsterdam, the Netherlands; b.lagerveld@slaz.nl 